\title{
Relationship of Stressful Childhood Experiences and Ability to Deal with Stress in Adulthood
}

DOMINICAN

UNIVERSITY of CALIFORNIA
Danielle Kinney

Psychology Department

Dominican University of California

\section{Introduction}

- People vary in past stressful childhood experiences and the ways they have learned to cope with day-to-day stress as adults. The childhood experiences influence adult reactions.

- Childhood stress has been shown to blunt cortisol response, lessening the adult stress response (Suzuki et al, 2014)

- Adults who had experienced significant stress as children more strongly reacted to small stressors in everyday life (Glaser, van Os, Portegijis and Myin-Germeys, 2006)

- Reactions to stress are offset by an individual's methods to cope. Studies show that there is indeed a specific association between methods of coping with childhood stress and current adjustment that is independent of a general association between disengagement methods of coping with almost any stressor. (Coffey et al, 1996).

- It was proposed that after experiencing so much childhood stress, an individual will react more intensely to stress in adulthood, but also have developed greater abilities to handle this, giving the individual a overall feeling of more control ove difficult life events.

\section{Hypotheses}

Hypothesis \#1: Individuals who experience a stressful childhood are more likely to perceive themselves as having greater ability to cope with more stress.

Hypothesis \#2: This perceived greater ability is specific and independent from generally higher self-esteem.

Hypothesis \#3 People who had a stressful childhood would be able to cope with stress better, so stressful events as adult will be perceived as less intense.

\section{Methods}

\section{Participants}

- 55 females, 8 males, 1 specified as "other" gender.

- Age range -18 to 64 years.

\section{Materials}

- Demographic Questions - gender and age only

- Childhood Trauma Scale - series of measures about possible occurrence of different types of events and level of trauma

- Perceived Stress Scale - two measures - level of stress experienced in the past week and significant event in past 3 years - Coping Self-Efficacy Scale - measures how well the participant thinks they cope with stress in adulthood

- Self-Esteem Scale - measure of participant self image

\section{Procedure}

- Questionnaires completed anonymously at an online survey site - Completion time: approximately 15 minutes

- Participants thanks and provided contact information to obtain general summary of results.

\section{Conclusions}

- Stressful Childhood Experiences:

- do not make a person become desensitized to adult stressors. - do not affect ability to cope with stress in adulthood.

- may result in perception of higher stress levels in response to events in adulthood.

- People who experience stressful childhoods have significantly lower self-esteem than those who did not have such experiences.

- Future research should consider coping styles to evaluate if childhood experiences influence how stress is managed.
Results

Group Comparison - Coping and Self-Esteem Scores Experienced Childhood Trauma $\stackrel{\text { YES }}{\text { Mean }(S D)} \quad \stackrel{\text { NO }}{\text { Mean }(S D)}$

t-test Mean $(S D) \quad$ Mean $(S D)$

Coping Score $131.6(30.5) \quad 137.8(33.7) \quad p=52 \%$

Self-Esteem $\quad 28.3(5.8) \quad 31.9(4.9) \quad p=2 \%$

Hypothesis \#1 was not supported. Participants who experience stressful childhoods were not different in their ability to cope with stress than those who did not have a stressful childhood.

Hypothesis \#2 was not supported. Participants who experience stressful childhoods had significantly lower self-esteem than those who did not have a stressful childhoods.

Group Comparison - Recent Perceived Levels of Stress Experienced Childhood Trauma YES NO Mean (SD) Mean (SD)

Perceived Stress: $71.3(22.2) \quad 63.3(29.3) \quad p=33 \%$ General Levels in the Past Week

Perceived Stress: $\quad 83.8(22.5) \quad 77.1(18.8) \quad p=32 \%$ During Significant

Event in past 3 years

Hypothesis \#3 was not supported - The group who had childhood trauma perceived the 3-year event with a higher amount of stress than those who did not have a stressful childhood. 